\title{
A Custom-made MATLAB Based Software to Manage Leakage Current Waveforms
}

\author{
Dionisios Pylarinos \\ High Voltage Lab \\ University of Patras \\ dpylarinos@yahoo.com
}

\begin{abstract}
Leakage current (LC) monitoring is a widely employed tool for monitoring the performance of high voltage insulators. LC waveform monitoring is required to acquire a view of the phenomena experienced since the type of $\mathrm{LC}$ waveform's shape is correlated with different types of electrical activity. In case of field monitoring, specially designed measuring systems, capable of operating under field conditions, have to be installed. The Greek Public Power Corporation (PPC) has issued a large project for monitoring the performance of outdoor insulators. A commercially available LC measuring system, was purchased and installed by PPC. The system was able to monitor various outdoor insulators, record several statistical values and intermittently record a $\mathrm{LC}$ waveform. The provided software lacked some capabilities that were required for certain research areas and a custom made software had to be developed. In this paper, two stand-alone MATLAB based softwares, "Leakage Current Waveform File Converter" and "Leakage Current Waveform Viewer", both equipped with a user friendly GUI, are presented. The first software was developed in order to automatically convert multiple files from the CSV format (as extracted from the measuring system) to the MAT format in order to allow data compactness and further processing. The latter software was developed in order to offer a tool for collective viewing, printing and extraction to JPEG format, of LC waveforms in groups of user-defined numbers. The latter function gives the opportunity for easy and fast creation of waveform archives.
\end{abstract}

Keyword: Leakage Current; Waveform; Data; Software; GUI; MATLAB

\section{INTRODUCTION}

Leakage Current (LC) is a widely applied tool for monitoring the electrical phenomena experienced on outdoor insulators. Leakage current waveform monitoring provides an actual view of the experienced phenomena since the shape of LC waveforms has been well correlated with different types of electrical activity. In addition, surface activity is strongly linked to environmental conditions and the accumulation and distribution of pollutants on the insulators' surface and, therefore, monitoring leakage current can be an indirect method for evaluating the pollution severity of an installation site. It should be noted that field measurements are most suitable since they offer an exact view of the experienced phenomena and of insulators' performance under real conditions. However, monitoring leakage current waveforms in the field is a rather complex task. The monitored insulators and the measuring system have to be installed in the field and to be subjected to various environmental and electrical stresses. Further, approaching the measuring system is also an issue since it requires entering an outdoor $\mathrm{HV}$ site. Therefore, specially designed measuring apparatuses that can operate unattended for long periods of time, under field conditions, have to be employed. Such systems inevitably face restrictions and limited capabilities compared to systems designed for laboratory use.

The Greek Public Power Corporation (PPC) has issued a large project to cope with the pollution problem in the case of Crete, a Greek island in the Mediterranean Sea [1-5]. As a part of this project a commercially available leakage current measuring system, On-line Leakage Current Analyzer (OLCA) by CTLAB [6], was purchased and installed at an $150 \mathrm{kV}$ High Voltage Substation. The system extracts and records a large amount of values calculated from the monitored LC and uses the time-window technique to intermittently record LC waveforms. The time-window technique allows the recording of a single leakage current waveform during a user-defined time window (e.g. one waveform per day) [7]. The system and the provided software was not oriented towards waveform monitoring and although it gave a large variety of functions related to extracted statistical amounts, it only allowed intermittently recording of waveforms and only a one by one viewing of recorded waveforms (the user can choose the starting and ending date). It also provided the ability to extract the measured waveforms to various CSV files. As the amount of recorded waveforms increased over the years of monitoring, the need of developing custom made software emerged. Two different stand-alone softwares were finally developed equipped with their own Graphical User Interface (GUI) in order to maintain a user-friendly environment that would allow their use even by not specialized PPC personnel.

\section{GOALS SET FOR THE DEVELOPED SOFTWARE}

The software's goals were to perform these basic functions:

1) conversion of multiple CSV files, that contained different measurements recorded on the same insulator through different time-windows, to a single MAT file representative of each insulator, that would allow further 
processing. To save time, all CSV files corresponding to different insulators should be converted sequentially.

2) navigation through LC waveforms, contained in a MAT file, in groups of waveforms of a user-defined number. The user should have the capability to choose a partial sum of the waveforms contained in the MAT file, using numerical or chronological criteria

3) automate the extraction of the various groups of waveforms to different JPEG files with user-defined names and dpi analysis

4) offer a user-friendly Graphical User Interface (GUI) which would allow ease of use even by non-specialized personnel.

5) stand-alone capability of files and ease of execution desirably by providing EXE files

\section{DEVELOPING ENVIRONMENT \& DEVELOPED SOFTWARE}

Two different softwares were developed using MATLAB, a high-level language and interactive environment manufactured by Mathworks [8]. To achieve the first goal, "Leakage Current Waveform File Converter" was developed. To achieve goals 2 and 3, "Leakage Current Waveform Viewer" was developed. Both softwares are equipped with a user-friendly GUI and are provided in stand-alone executable formats (EXE) (goals 4 and 5)

\section{LEAKAGE CURRENT WAVEFORM FILE CONVERTER}

\section{A. Name pattern of CSV files}

The measuring system stored all measurements in a not disclosed format that was used internally by the provided software. The provided software however was able to extract the measured waveforms in CSV files. It should be noted that multiple CSV files were created with each conversion, with some of them being null. All CSV files followed the same pattern regarding their name as shown in Figure 1. All files were named "olca $\mathrm{X}_{1}$ PeakWaveformsChan $\mathrm{X}_{2} \mathrm{CX}_{3}$.csv" with $\mathrm{X}_{1}$ being the device model number, $X_{2}$ being the number of the monitored channel (one channel for each monitored insulator) and $\mathrm{X}_{3}$ being a numerical value. The value $\mathrm{X}_{3}$ corresponded to the actual number of files minus one, since the first file had a zero value of $X_{3}$. The files shown in Figure 1 with $1 \mathrm{~KB}$ size are null. The provided software created several such files when exporting measured waveforms to CSV files. Further, it should be noted that the number of extracted files differ in each case and the manufacturer did not provide any information for $\mathrm{X}_{3}$ or for the creation of null files.

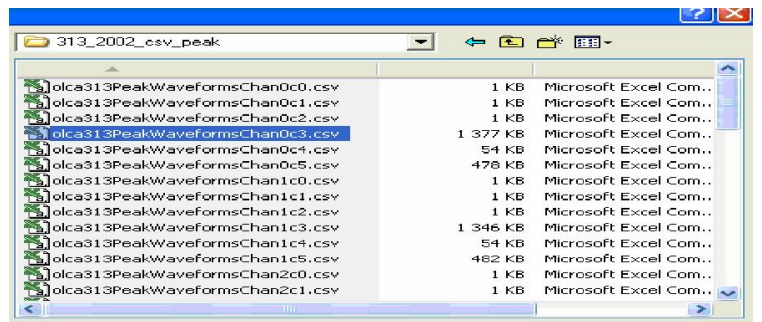

Fig. 1. A screenshot from a folder containing CSV files as extracted by the measuring system

\section{B. Software GUI and user settings}

The Leakage Current Waveform File Converter took into account the format of the CVS files and provided a GUI where the user could define each value, as shown in Figure 2. In addition, the user could decide if he wanted to convert just one CSV file or perform batch conversion. The user could also choose to sequentially convert files corresponding to more than one channel. In case of batch conversion the user should set the number of the OLCA model, the maximum value of $\mathrm{X}_{3}$, the directory where the CSV files are located, the number of channels and finally the destination directory where the MAT files should be stored.

\section{Converting files}

After the user defined all factors, the conversion would start when hitting the "CONVERT" button. This procedure could be extremely time consuming so a "PROCESS INFO" button was added in order to give a view of the procedure and offer a double-check opportunity before converting. The message displayed when pressing the "PROCESS INFO" button is shown in Figure 3.

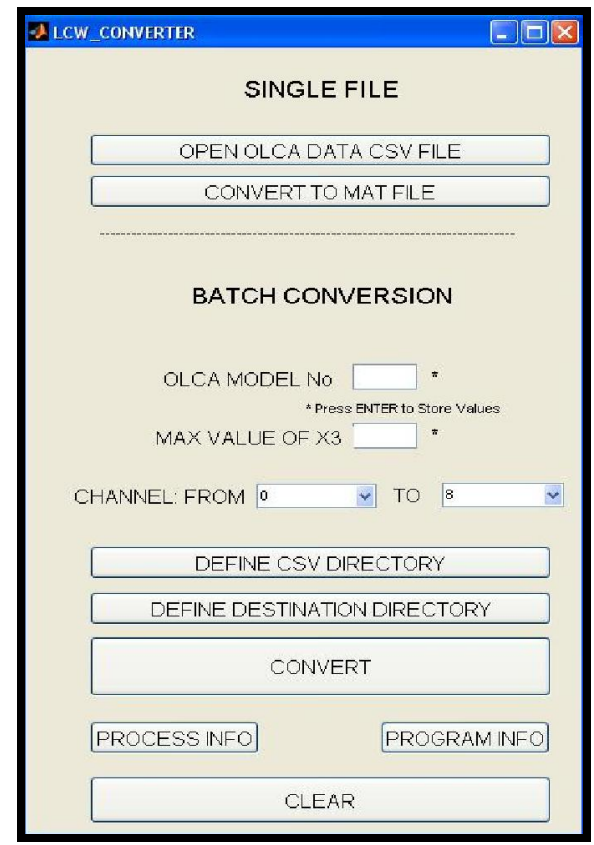

Fig. 2. Leakage Current Waveform File Converter

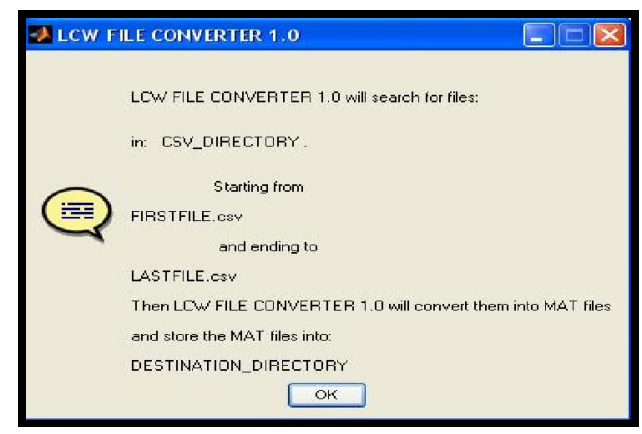

Fig. 3. An example of pressing the "PROCESS INFO" button 


\section{Program info}

A "PROGRAM INFO" button was added in order to provide users with some information about the software as shown in Figure 4. It should also be noted that when performing the conversion, a message appears that informs the user about the file the number of waveforms contained in the CSV file that is currently converted and finally for the path containing the MAT file.

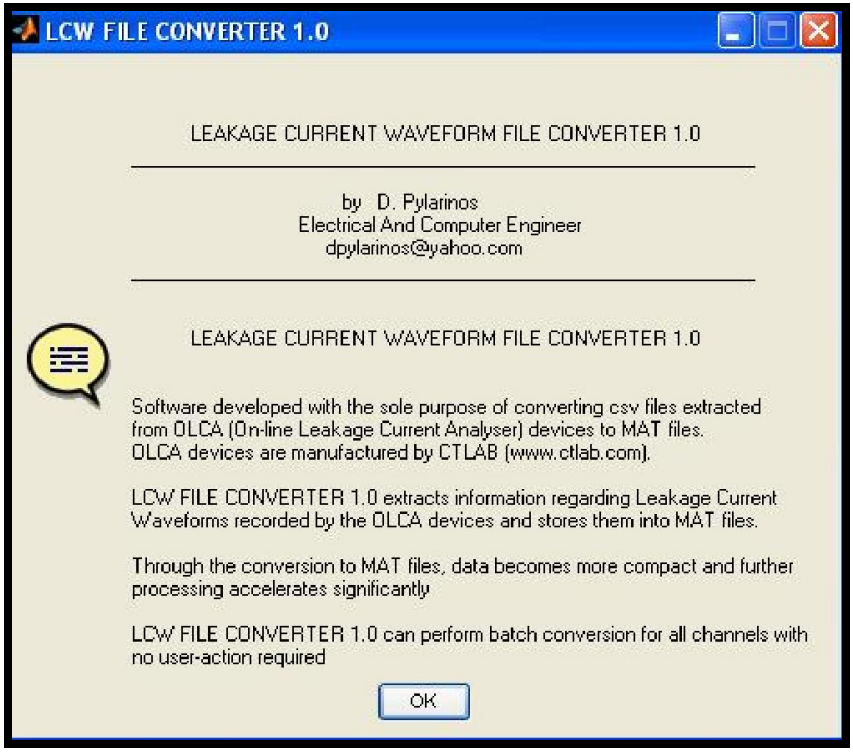

Fig. 4. The message displayed when pressing "PROGRAM INFO"

\section{E. Format of CSV files}

The software takes under consideration the format of the CSV files which contain waveforms of 960 data points [7] as shown in Figure 5. The first 5 rows of each file were occupied by general data and an empty line is inserted between different waveforms as shown in Figure 5.

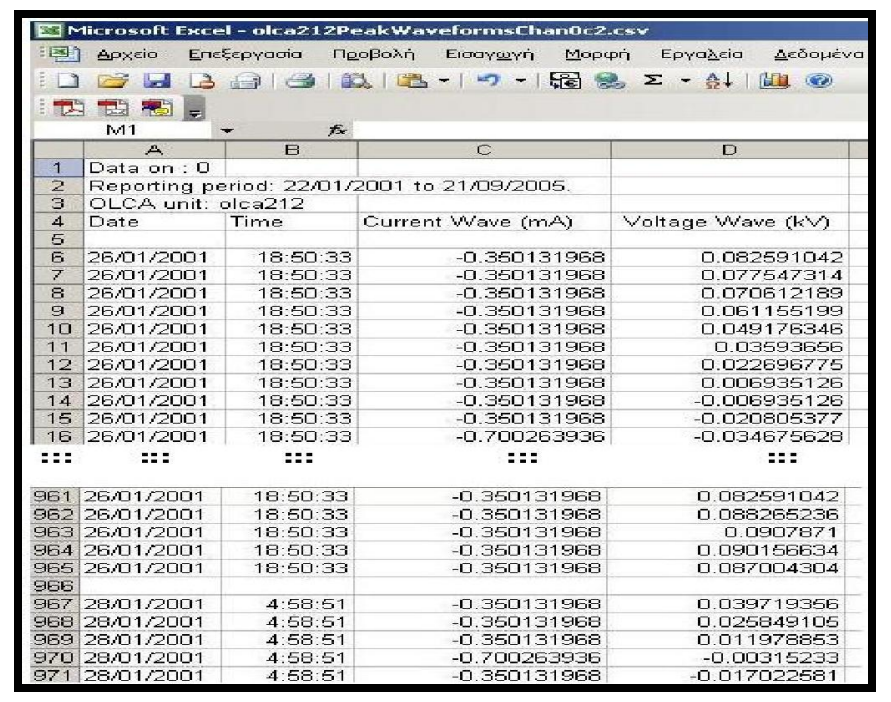

Fig. 5. Some of the data in a CSV file

\section{F. Format and variables of each MAT file}

The "Leakage Current Waveform File Converter" creates a MAT file for all measurements regarding the same channel (insulator) (e.g. olca313ch0) based on the information provided by the user in paragraph IV-b. Each MAT file contains the measurements in a much more organized format. In addition, it allows the application of various signal processing techniques as employed in $[4,7,9]$. Figure 6 shows the variables in a MAT file that contains 7497 waveforms, as portrayed by MATLAB when using the "whos" command. As shown, the variables include two vectors that contain date and time information about each measurement and two matrices ("current" and "voltage") that contain all current and voltage waveforms, with each waveform placed in an individual column. In addition, an "id" variable characterizes the sum of the waveforms and an "idwavetag" vector contains an individual name tag for each waveforms. The new form of the organized data gives the user far better viewing, managing and processing abilities compared to the CSV version.

\begin{tabular}{|c|c|c|c|c|}
\hline \multicolumn{5}{|c|}{ Command Window } \\
\hline \multicolumn{5}{|c|}{ (1) New to MATLAE? Watch this Video, see Demos, or read Getting Statted. } \\
\hline \multicolumn{5}{|l|}{$\gg$ whos } \\
\hline Name & size & Bỵtes & Class & Attributes \\
\hline current & $960 \times 7497$ & 57576960 & double & \\
\hline date & $1 \times 7497$ & 599760 & cell & \\
\hline id & $1 \times 5$ & 10 & char & global \\
\hline Idwavetag & $1 \times 7497$ & 702504 & cell & \\
\hline time & $1 \times 7497$ & 569772 & cell & \\
\hline voltage & $960 \times 7497$ & 57576960 & double & \\
\hline
\end{tabular}

Fig. 6. Variables contained in a MAT file

\section{G. Economy of space and compactness of MAT files}

It should be noted that a significant save of space and a much more compact data appearance was achieved file-wise. Each OLCA device monitors nine channels (numbered 0 to 8). All measurements from each channel were contained in a single MAT file instead of numerous CSV files. Results of using the Leakage Current Waveform File Converter on CSV files that were exported from two different OLCA devices are shown in Table 1. The data now was included in just 18 files (instead of 477) and a significant save of space was achieved (from 2.92 GB to $32.75 \mathrm{MB}$ )

TABLE I. RESULTS OF USING LEAKAGE CURRENT WAVEFORM FILE CONVERTER

\begin{tabular}{|c|c|c|c|}
\hline OLCA DEVICE & $\mathbf{1}$ & $\mathbf{2}$ & Sum \\
\hline Number of CSV files & 225 & 252 & 477 \\
\hline Size of CSV files & $350.6 \mathrm{MB}$ & $2.57 \mathrm{~GB}$ & $2.92 \mathrm{~GB}$ \\
\hline Number of MAT files & 9 & 9 & 18 \\
\hline Size of MAT files & $3.85 \mathrm{MB}$ & $28.9 \mathrm{MB}$ & $32.75 \mathrm{MB}$ \\
\hline $\begin{array}{c}\text { Total number of } \\
\text { waveforms }\end{array}$ & 8487 & 67377 & 75864 \\
\hline
\end{tabular}




\section{LEAKAGE CURRENT WAVEFORM VIEWER}

The MAT files created by "Leakage Current Waveform File Converter" can then be loaded into the "Leakage Current Waveform Viewer". The "Leakage Current Waveform Viewer" was developed to satisfy goals 2 and 3 mentioned in paragraph II. The GUI of the software if shown in Figure 7.

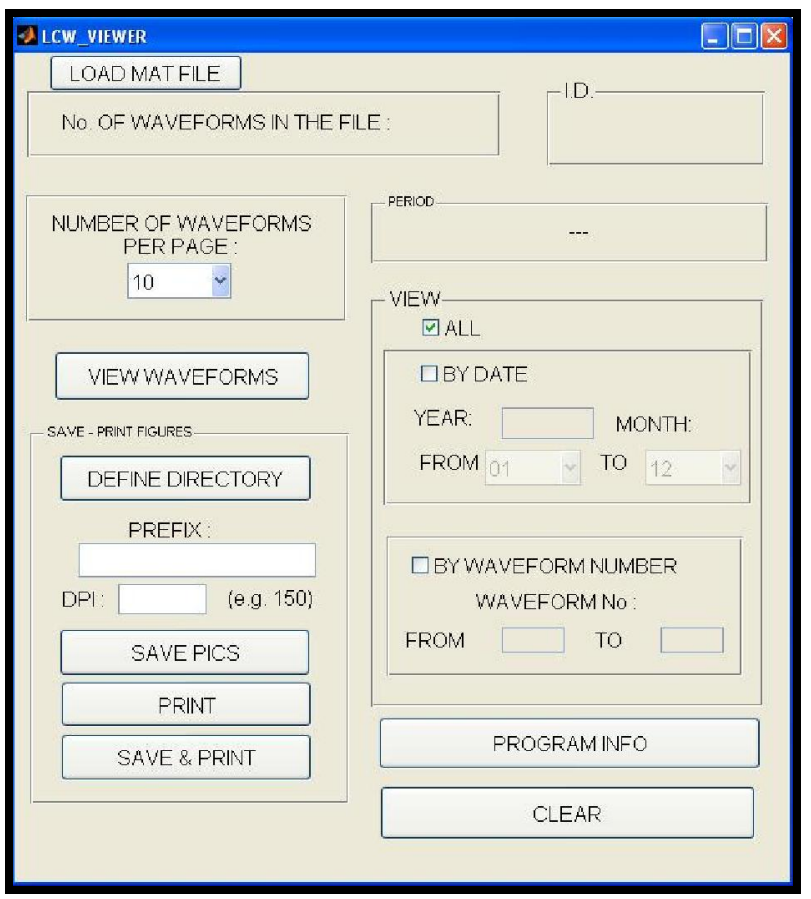

Fig. 7. Leakage Current Waveform Viewer

\section{A. Loading a MAT file}

At first the user has to load the MAT file created by "Leakage Current Waveform File Converter". It is obvious that using MATLAB one can alter (e.g. delete data correlated with certain waveforms) or unite different MAT files. When a MAT file is loaded, "Leakage Current Waveform Viewer" shows the number of waveforms that are contained in the file, the "id" variable that characterizes the group of waveforms (set by the user, may represent the type of the monitored insulator, the channel number combined with the OLCA model etc) and the time period that these measurements cover. An example is given in Figure 8, when loading the MAT file already mentioned in paragraph IV-F. "Leakage Current Waveform Viewer" now shows to the user the number of waveforms contained in the file (7497), the "id" variable (in this example it is set by the user to be "compB" which characterizes a monitored composite insulator), and the time period (from $26 / 11 / 2002$ to $06 / 08 / 2008$ ). To define the time period, "Leakage Current Waveform Viewer" uses the first and last value of the "date" variable (see paragraph IV).

\section{B. Grouping}

The user is now ready to view, extract or print the waveforms contained in the file. At first, he should decide upon the group of waveform that he wants to manipulate. In the area with the heading "VIEW" he can choose whether he wants to view all waveforms or just a part of them, defining either their number (e.g. from waveform number 556 to waveform number 2147) or the time period that they cover (by defining the year and the starting and ending month). The waveforms will be displayed in groups and the next step for the user is to define the number of waveforms in the group. The default value is 10 but several other values can be selected $(1,4,6,8,10, \ldots, 18,20)$.

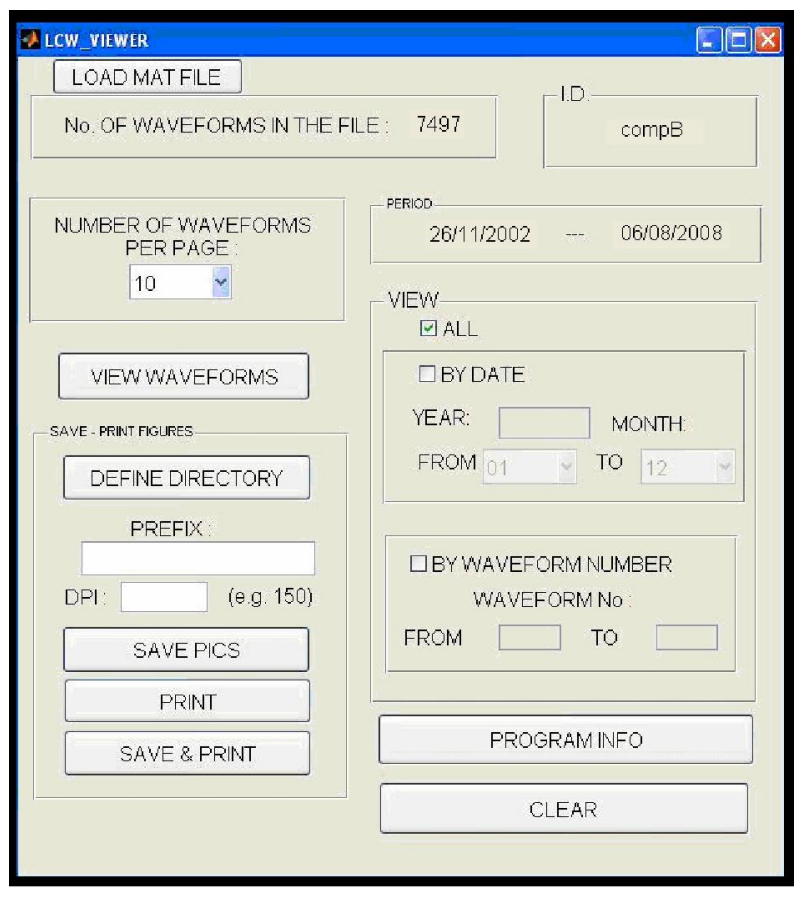

Fig. 8. Leakage Current Waveform Viewer (MAT loaded)

\section{Viewing leakage current waveforms}

To view the waveforms the user presses the "VIEW WAVEFORMS" button. Then, "Leakage Current Waveform Viewer" opens a new window as shown in Figure 9. In this figure, the measurements portrayed were provided by the manufacturer with the purchase of the system.

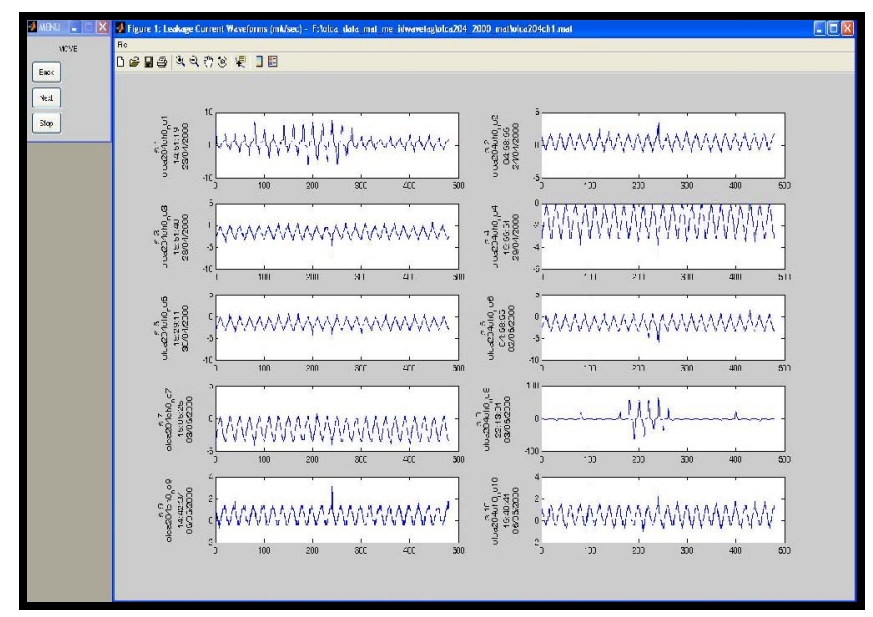

Fig. 9. The VIEW WAVEFORMS window (groups of ten) 


\section{The navigation menu}

The navigation menu is located at the top left of the window. A zoom-in version of the navigation menu is shown in Figure $10 \mathrm{a}$. The user can move to the next or previous group or stop. When he reaches the last group (in this case the last ten waveforms) then the navigation menu changes to the one shown in Figure 10b giving him the opportunity to move to the first waveforms.

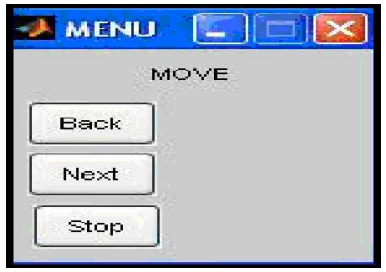

(a)

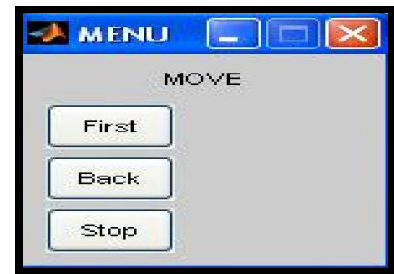

(b)
Fig. 10. The navigation menu: a) normal b) at the last group

\section{E. The main VIEW window}

The main VIEW window (the window that contains the waveforms) includes various informations. The window title contains the kind of measurement, the axis units and the path of the filename that contains the waveforms. A zoom in of the window title is shown in Figure 11. Further, some of the usual image managing buttons (move, save as, zoom etc) are also available.

In addition, to the left of each waveform appear its place in the group (s. 1 stands for signal 1), their unique id tag (contained in the variable "idwavetag") which contains the OLCA model, the channel and the number of the waveform in the MAT file, the time when the waveform was recorded and the date. The "idwavetag" may seem redundant in the case of Figure 11 but one should consider the possibility of choosing to view e.g. waveforms 75 to 154 . In this case the first waveform (waveform 75) will have the first place in the selected group (s.1) but its" idwavetag "will portray its actual place in the file (75). In addition, using MATLAB the user can easily unite two MAT files that contain waveforms from different channels and load them into "Leakage Current Waveform Viewer". The "idwavetag" will also prove valuable in this case. An example is shown in Figure 12.

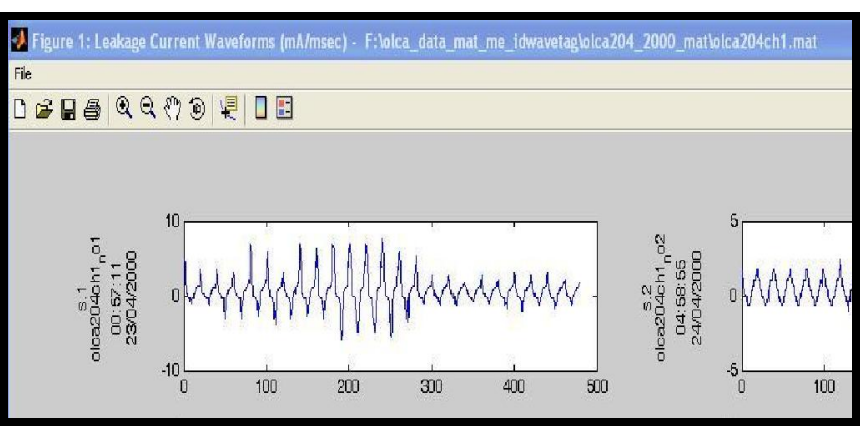

Fig. 11. VIEW window. detail of the window title

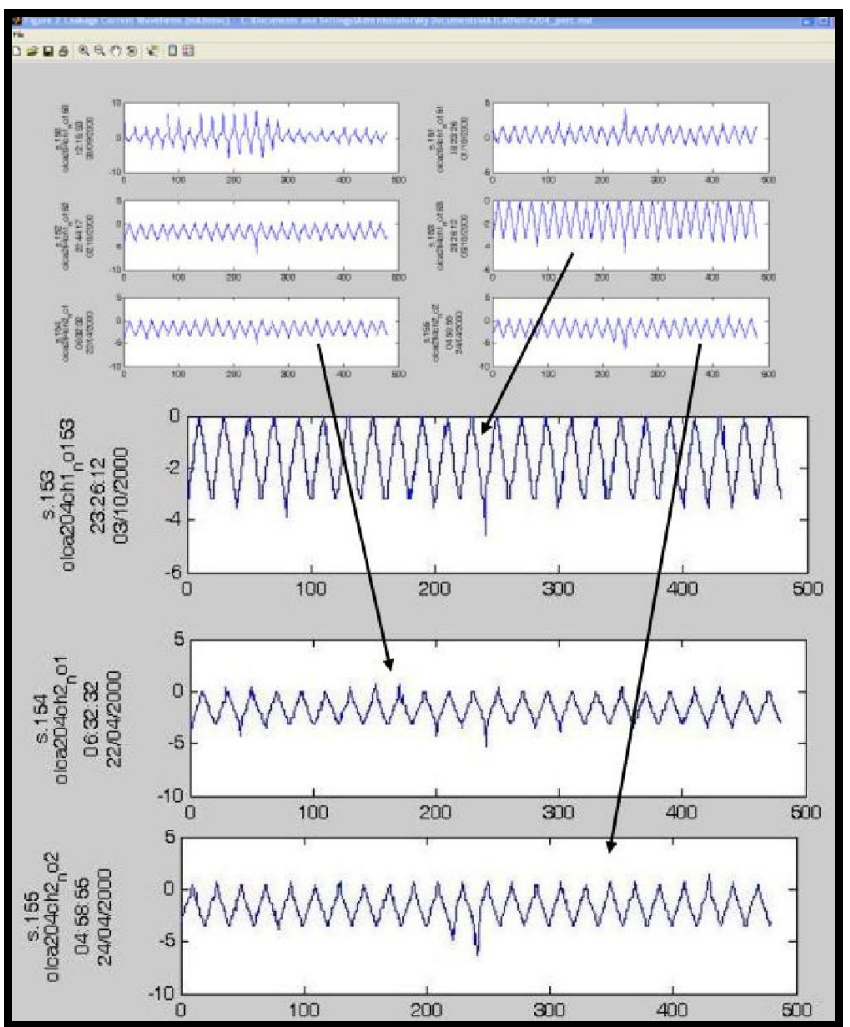

Fig. 12. Viewing measurements recorded in different channels (zoom in)

\section{F. Exporting \& printing leakage current waveforms}

The images in the VIEW WAVEFORMS window can be automatically printed or exported to JPEG image files. In case of exporting, the user has to define the destination folder for the JPEG files by pressing the "DEFINE DIRECTORY" button. Each group of waveforms will be printed in one page and/or exported in one JPEG file. That means that if a set of 120 waveforms is chosen to be displayed in group of 10 waveforms, then 12 JPEG image files will be created. The first image will contain waveforms 1-10, the second will contain waveforms 11-20 and so on. The user has to provide a prefix for the name of the JPEG files. For example if the user enters the prefix "images" then the first JPEG image file will be named "images_0001", the second will be named "images_0002" and so on. The user can also define the dotsper-inch (dpi) analysis of the images. Finally the user can choose to print or export or do both by pressing the corresponding buttons ("PRINT", "SAVE", "SAVE \& PRINT").

\section{G. Process info}

The exporting and/or printing procedure can be time consuming. For example, when a MAT file containing more than 7000 waveforms is loaded in the software, as the one showed in Figure 8, and a grouping of 10 is chosen, this will result to the creation of more than 700 JPEG files. Needless to say that the user should be sure about his settings before the procedure starts. Therefore a question message has been added 
as shown in Figure 13. The message is different for each case ("SAVE", "PRINT" or "SAVE \& PRINT" of waveforms).

(a)

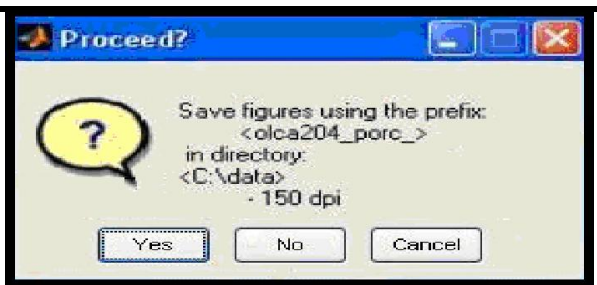

(b)

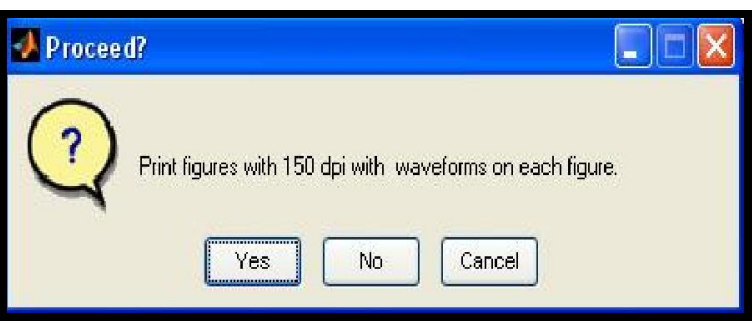

A Proceed?

(c)

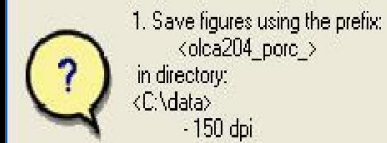

2. Print figures with 150 dpi with 10 waveforms on each figure.

\section{Yes No Cancel}

Fig. 13. The "Proceed" message in case of a)exporting to JPEG b) printing to paper c) exporting \& printing

\section{H. Program info}

The "PROGRAM INFO" offers some information about the software. The message displayed when pressing the "PROGRAM INFO" button is shown in Figure 14.

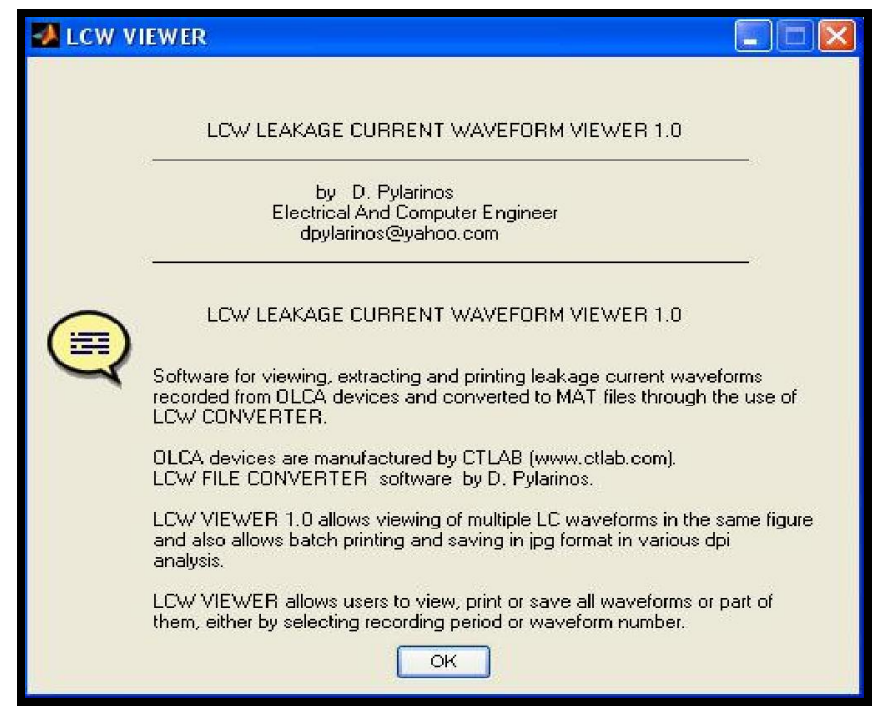

Fig. 14. The message displayed when pressing "PROGRAM INFO"

\section{CONCLUSIONS}

Leakage current monitoring is a well established tool for monitoring outdoor high voltage insulators in the field. However, specially measuring designed systems have to be installed capable of operating unattended under real field conditions. The Greek Public Power Corporation purchased such systems as a part of a large scale project, to investigate and battle with the pollution of high voltage insulators in the Cretan Transmission system. In this paper, two stand-alone MATLAB based softwares that were developed in order to offer some custom capabilities that the provided software lacked, are presented.

"Leakage Current Waveform File Converter" was developed to convert CSV files, containing measurements exported from the measuring system, to MAT files that would allow further processing. Further, the software provided data in a much more compact form and allowed a significant save of disk space.

"Leakage Current Waveform Viewer" was designed to manage the MAT files created by "Leakage Current Waveform File Converter". The software is able to divide the data in userdefined groups and display each group sequentially, providing a navigation menu. The user can also decide to manipulate only a part of the data-set using numerical or chronological criteria. The software provides the capability of automatically exporting all waveform groups to different JPEG files named using a user-defined prefix and an automatically assigned numerical value. The user can set the desired dpi analysis. Finally, the software can automatically print images without exporting them, or export them and print them to paper at the same time.

Both softwares are equipped with user friendly GUI's that allows their use by non-specialized personnel and are distributed in EXE format. They are currently employed to extend and enhance the research and investigation of field leakage current waveforms.

\section{REFERENCES}

[1] K. Siderakis, D. Pylarinos, E. Thalassinakis, I. Vitellas, E. Pyrgioti, "Pollution maintenance techniques in coastal high voltage installations", Eng. Technol. Appl. Sci. Res., Vol. 1, No. 1, pp. 1-7, 2011

[2] K. Siderakis, D. Agoris, "Performance of RTV silicone rubber coatings installed in coastal systems", Electr. Power Syst. Res., Vol. 78, No. 2, pp. $248-254,2008$.

[3] D. Pylarinos, S. Lazarou, G. Marmidis, E. Pyrgioti, E. Thalassinakis, J. Stefanakis, "Investigation of leakage current on high voltage polymer (RTV SIR) coated insulators", IASTED Int. Conf. on Power and Energy Systems, USA, 2008

[4] D. Pylarinos, S. Lazarou, G. Marmidis, E. Pyrgioti, "Classification of surface condition of polymer coated insulators using wavelet transform and neural networks", ICWAPR Int. Conf. on Wavelet Analysis and Pattern Recognition, pp. 658-663, China, 2007

[5] E. Thalassinakis, J. Stefanakis, K. Siderakis, D. Agoris, "Measures and techniques against pollution in the Cretan transmission system", EuroPES European Power and Energy Systems, GREECE, 2002

[6] CTLAB Electronic Development Manufacturing, http://www.ctlab.com/

[7] D. Pylarinos, K. Siderakis, E. Pyrgioti, E. Thalassinakis, I. Vitellas, "Impact of noise related waveforms on long term field leakage current measurements", IEEE Trans. Dielectr. Electr. Insul., Vol. 18, No. 1, pp. $122-129,2011$

[8] Mathworks web page: http://www.mathworks.com/ 
[9] D. Pylarinos, K. Siderakis, E. Pyrgioti, E. Thalassinakis, I. Vitellas, "Automating the classification of field leakage current waveforms", Eng. Technol. Appl. Sci. Res., Vol. 1, No. 1, pp. 8-12, 2011

\section{AUTHORS PROFILE}

Dionisios Pylarinos was born in Athens in 1981. He received a Diploma degree in Electrical and Computer Engineering from the University of Patras in 2007. Presently he is with the High Voltage Laboratory of the Department of Electrical and Computer Engineering at the University of Patras. He has worked as a scientific consultant for the Greek Public Power Corporation (PPC). His research interests include outdoor insulation, electrical discharges, signal processing and pattern recognition. 